\title{
Colour text segmentation in web images based on human perception
}

\author{
D. Karatzas ${ }^{a}$, A. Antonacopoulos b,* \\ ${ }^{a}$ School of Electronics and Computer Science, University of Southampton, Southampton SO17 1BJ, United Kingdom \\ ${ }^{\mathrm{b}}$ Pattern Recognition and Image Analysis (PRImA) Research Lab, School of Computing, Science and Engineering University of Salford, \\ Manchester M5 4WT, United Kingdom
}

Received 24 July 2004; received in revised form 14 April 2006; accepted 16 May 2006

\begin{abstract}
There is a significant need to extract and analyse the text in images on Web documents, for effective indexing, semantic analysis and even presentation by non-visual means (e.g., audio). This paper argues that the challenging segmentation stage for such images benefits from a human perspective of colour perception in preference to RGB colour space analysis. The proposed approach enables the segmentation of text in complex situations such as in the presence of varying colour and texture (characters and background). More precisely, characters are segmented as distinct regions with separate chromaticity and/or lightness by performing a layer decomposition of the image. The method described here is a result of the authors' systematic approach to approximate the human colour perception characteristics for the identification of character regions. In this instance, the image is decomposed by performing histogram analysis of Hue and Lightness in the HLS colour space and merging using information on human discrimination of wavelength and luminance.
\end{abstract}

(C) 2006 Elsevier B.V. All rights reserved.

Keywords: Web document image analysis; Colour document analysis; Character segmentation; Text segmentation; Colour images

\section{Introduction}

Images constitute an important part of the information content of any document. In the case of Web documents, in particular, images play a crucial role in bringing visual impact to an otherwise plain text medium. In addition to illustrations, Web document designers also frequently create text in image form on Web pages, as an attempt to overcome the stylistic limitations of HTML.

The text that is created in image form corresponds more often than not to document headers, titles, links to sections or banners and, therefore, has a potentially high semantic value. One common use of this semantic value is in terms of indexing and ranking the Web pages that contain this text. For instance, a search term contained within a document title is likely to indicate that

\footnotetext{
* Corresponding author.

E-mail address: A.Antonacopoulos@primaresearch.org Antonacopoulos).

$U R L:$ http://www.primaresearch.org (A. Antonacopoulos).
}

the document is more relevant than another document in which the same term was found somewhere towards the end of its main body.

The situation can be worse if the text in image form is not found at all. As current search engine technology does not allow for text extraction and recognition in images (see [1] for a list of indexing and ranking criteria for different search engines), the text in image form is ignored.

Ignoring (not being able to access) the text embedded in images can be a serious matter since this information may not be present anywhere else in the document. According to a study carried out by the authors [2], of the total number of words visible on a Web page, $17 \%$ are in image form (most often semantically important text). Worse still, 76\% of these words in image form do not appear elsewhere in the encoded (e.g., ASCII or UNICODE) text. These results agree with earlier findings [3] and clearly indicate an alarming situation that does not seem to be improving.

Another significantly desirable goal is to obtain a uniform representation (e.g., UNICODE) of all visible text (whether in image form or not) in a Web document. This 
uniform representation can be used in a number of applications such as translation, summarisation, voice browsing [4] and automated content analysis [5] for rendering (reflowing) on small screen devices such as PDAs and mobile phones. A number of further applications are emerging in the field of Web Document Analysis [6]. The extraction and recognition of text in image form is a significant step towards achieving this representation.

There has been a formal provision for specifying the encoded (ASCII/UNICODE) equivalent of the text in Web images, in the form of ALT tags in HTML. However, the same study mentioned earlier [2], assessing the impact and consequences of text contained in images, indicates that the ALT tag strategy is not effective. It was found that the textual description (ALT tags) of $56 \%$ of images on Web pages was incomplete, wrong or did not exist at all.

It can be seen from the above that there is a significant need for methods to locate and recognise the text in images in Web documents. Considering traditional optical character recognition (OCR), one may initially think that Web images present some advantages over scanned documents, such as the lack of digitisation-induced noise and skew. However, the task is considerably difficult for traditional OCR for a number of reasons. First, these (often complex) colour images tend to be of low resolution (usually just $72 \mathrm{dpi}$ ) and the font size used for text is very small (about 5-7pt). Such conditions clearly pose a challenge to traditional OCR, which works with $300 \mathrm{dpi}$ images (typically bilevel) and character sizes of usually $10 \mathrm{pt}$ or larger. Moreover, images on Web documents tend to have various artefacts introduced by the authoring software (e.g., by performing colour quantization and lossy compression) [3]. A comparison of the characteristics faced by the task of analysing text in Web images versus the input expected by traditional OCR can be seen in Table 1.
Without loss of generality, the goal of achieving an encoded representation of the text in image form can be split into three objectives (and corresponding stages):

a. Character segmentation. The image must be segmented first so that regions corresponding to potential character components are separated from the background. A successful segmentation will be one where background and foreground regions are not merged.

b. Text extraction. Character-like components that fulfil criteria of constituting text (e.g., they appear to form a textline) are extracted.

c. Text recognition. Strings of character-like components are recognised, possibly aided by application or document-specific information.

In view of the difficulties posed by the image and text characteristics (as faced by traditional document analysis methods) it can be appreciated that the segmentation stage is by far the most challenging. It is also the most crucial in terms of the detrimental effect of any errors made at that stage. As such, it merits special attention and individual study.

This paper presents a new approach for character segmentation, especially in complex Web images (e.g., see Fig. 1). It argues that the RGB colour space representation (commonly used by previous approaches - see below) is not suited to this particular task and adopts a segmentation method based on analysing differences in chromaticity and lightness that are closer to how humans perceive distinct objects. This is the authors' first approach among a number of alternatives in their pursuit of different ways to address this problem by exploiting human colour perception. An initial account of work in progress of this method has been presented in [7]. This paper constitutes the entire and detailed account of the now completed and systematically evaluated approach.

Table 1

Characteristics of text in web images versus scanned documents

\begin{tabular}{lll}
\hline Characteristics & Text in image form $(\mathrm{web})$ & Typical scanned document images \\
\hline Resolution & approx. 72 dpi & $\geqslant 300 \mathrm{dpi}$ \\
Image size & $100 \mathrm{~s}$ of pixels & $1000 \mathrm{~s}$ of pixels \\
Character size & Can be as small as 5-7pt & $\geqslant 10 \mathrm{pt}$ \\
Colour scheme & Multi-colour text over multicolour background & Monochrome text over monochrome background (usually black/white) \\
Artefacts & Anti-aliasing, lossy compression, colour dithering & Skew, digitisation artefacts \\
Character effects & Characters not always on a straight line, 3D-effects, & Characters usually on a straight line, of the same font \\
& shadows, outlines etc. &
\end{tabular}

a

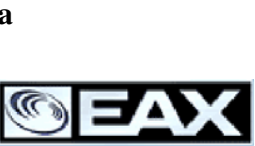

b

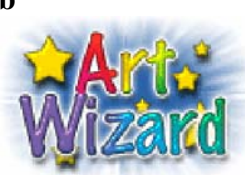

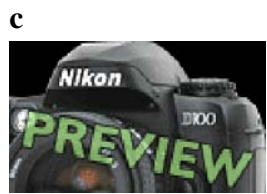

d

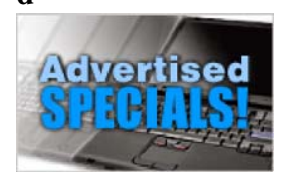

e

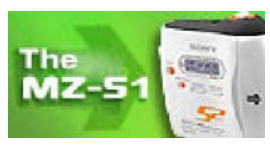

Fig. 1. (a and b) Images containing gradient text. (c) Image with transparent text over photographic background. (d) Image with single colour text over photographic background. (e) Image containing single colour text over multicolour background. 
In the following section, relevant previous work is briefly reviewed. In Section 3, the rationale of the whole approach and details of the different stages of the text segmentation method are described. Experimental results are presented and evaluated in Section 4. A brief description of a relevant extension in the form of text extraction is given in Section 5 before the paper concludes in Section 6.

\section{Related work}

Responding to the evident need for text recovery from Web images, a small number of approaches have been proposed. Zhou and Lopresti [3] have proposed methods for text segmentation, extraction and recognition. Their method for text segmentation and extraction is based on clustering in the RGB colour space and then for each cluster assessing connected components whose colour belongs to that cluster. The approach works well with GIF images (only 256 colours) and when characters are of almost uniform (constant) colour. With similar assumptions about the colour of characters, the segmentation approach of Antonacopoulos and Delporte [8] uses two alternative clustering approaches in the RGB space but works on (bit-reduced) full-colour images (JPEG) as well as GIFs. Jain and $\mathrm{Yu}$ [9] report a segmentation method based on decomposing an original image into a number of foreground images and a background one. The original number of colours (8-bit or 24-bit images) is dramatically reduced (to between 4 and 8 distinct colours) by bit dropping and colour quantization in the RGB space. Although this method produces good results for relatively simple images, it shares the same problems with the other methods when more complex images are encountered.

Existing approaches assume a practically constant and uniform colour for text and fail when this is not the case. In practice, there are many situations where gradient or multicolour text is present. Furthermore, the background may also be complex (in terms of colour) so that the assumption that it is the largest area of (almost) uniform colour in the image [9] does not necessarily hold.

For completeness, it should be mentioned that the problem of text recovery from Web images is by no means equivalent to the separate problems of recovering text from video sequences or real scenes, although it may appear so at a superficial level. The recovery of text from video (e.g., captions, credits) takes advantage of certain characteristics of video that are either not possible or not practical to assume for text in Web images. Such characteristics include the fact that text remains between frames or scrolls independently of the rest of the data [10] and that text is of uniform colour and appears on horizontal textlines [11].

With regard to the recovery of text from natural scenes (including scene text in video), there are also specific application-domain characteristics that do not generally apply to the recovery of text from Web images. On one hand, scene text can be unevenly illuminated and suffer from 3-D projection distortions. On the other hand, existing methods are based on the assumptions that the text is of uniform colour (as well as its immediate background), that text originally exists in horizontal textlines and that there is high contrast between text and background in the image/ frame [12-14].

\section{The method}

The proposed method is designed to exploit characteristics of the human perception of colour differences, based on certain observations about Web image text. The most important observation is that text in Web Images is produced using only software (no alterations occur by printing/scanning) and optimised for viewing on a monitor screen. This fact manifests itself in a number of ways. Apart from the issues listed in Table 1, the focus at this point is on the design process of Web image text. As this text (headers, banners etc.) is created in image form to add impact to the message of the document, the colour of the text and its visual distinction from the background is chosen (consciously or subconsciously) according to how humans perceive it to 'stand out'.

The premise of this paper is that a method for text extraction in these circumstances will benefit from the analysis of chromaticity and lightness differences as humans perceive them and not necessarily as expressed in the RGB space. In reality, colours that have equal distances in the RGB space are perceived by humans as having unequal differences ${ }^{1}$. Therefore, the difference between colours that were designed to be contrasting will be perceived by humans as disproportionately greater than that between other colours, whereas the pairwise distances in the RGB space may not be as indicative of the colour difference. Similarly, colours belonging to the same object will be designed to be perceived as more similar (irrespective of their RGB distance).

The approach presented in this paper is based on a combination of the Hue-Lightness-Saturation (HLS) representation of computer colour and biological data that describes the means by which humans differentiate between colours of different wavelengths, colour purities and luminance values. The rationale behind the selection of the HLS colour system, as well as the set of biological data used, will be described in the next section.

In overview, the method works in a split-and-merge manner, aiming to identify and analyse regions that are perceptually different in colour. The image is first (recursively) split, based on histogram analysis, and a tree structure of layers is created. Connected components are then identified in the leaf layers of the tree structure. Subse-

\footnotetext{
1 For example, assume that two colours have RGB (Euclidian) distance $\delta$. Humans find it more difficult to differentiate between the two colours if they both lie in the green band than if the two colours lie in the red-orange band (with the distance remaining $\delta$ in both cases). This is because humans are more sensitive to the red-orange wavelengths than they are to the green ones.
} 
quently, a merging process combines connected components of similar colour and satisfactory interrelationship (to progressively assemble characters) in a bottom up fashion, starting in the leaf layers, and moving up to the root layer of the tree.

\subsection{Human colour perception and discrimination}

Colour is expressed by measurements of its physical properties: wavelength, colour purity and luminance. These properties give rise to the psychophysical attributes of hue, saturation and lightness, which constitute a description of colour as perceived by humans. The approach described in this paper has been designed so that, both the colour system employed and the way of expressing and evaluating colour differences are directly related to this description of the human perception of colour.

The choice of colour system has been influenced by the observation that the use of perceptually oriented quantities (the psychophysical attributes described above) can prove more suitable for the analysis of images created to be viewed by humans $[15,16]$ than RGB components (which are used in computer systems to represent colour). Each of the components of the HLS colour system (Hue, Lightness and Saturation), which is used here, is directly related to the corresponding psychophysical attribute (hue, lightness and saturation).

Another important consideration in the design of the approach described here is the expression of the ability of humans to distinguish between colours. This ability is affected by numerous factors primarily wavelength separation (i.e., distance between given wavelengths), colour purity and lightness [17]. As wavelength separation (between colours) increases, the ability to discriminate between colours increases accordingly. Increases in colour purity maximize the perceived distance between colours. Finally, humans are able to differentiate between colours with higher lightness values much more easily than between those with lower lightness values.

The ability of the HLS colour system to express the above factors is straightforward. The $H$ (Hue) component of the HLS system corresponds to the perceived wavelength of each colour, thus larger differences in Hue are translated to better wavelength separation. The $S$ (Saturation) component corresponds to colour purity, while the $L$ (Lightness) component corresponds to lightness. Based on the above discussion, the case for using the HLS colour system for colour analysis of Web images is further strengthened.

In order to characterise whether two colours are perceived as similar or not, based on given HLS values, the representation of colour in HLS must be coupled with information about the intrinsic thresholds of human vision (i.e., the least noticeable differences that a human can perceive in Hue, Lightness and Saturation).

Certain biological information on colour discrimination (by humans) has been published in terms of expressions of

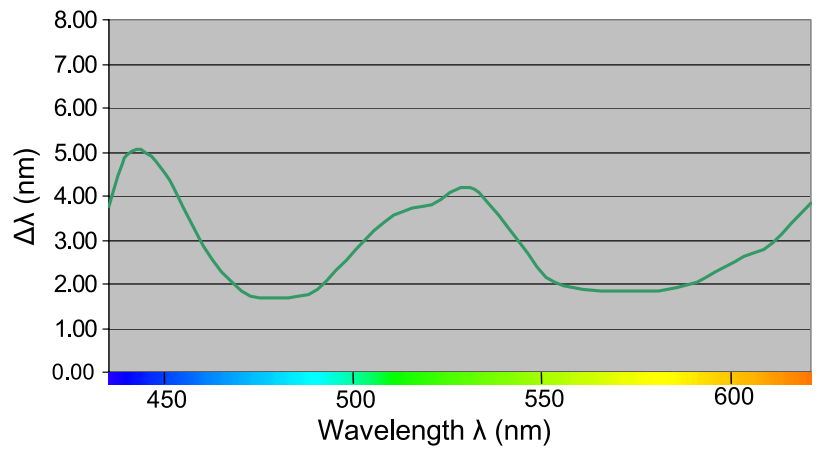

Fig. 2. The average change in wavelength required for a perceivable difference in hue to appear.

minimum discrimination ability as a function of each of the physical properties of colour (wavelength, colour purity and luminance as mentioned in the beginning of this section) $[18,19]$. The diagram in Fig. 2 illustrates the change in wavelength required to produce a perceived change in hue.

Experiments were designed and conducted by the authors in order to establish whether existing colour discrimination information could be used in the specific application domain (colour discrimination between small-sized coloured areas viewed under largely uncontrolled everyday viewing conditions). The measured thresholds corresponded well to the published biological information, verifying thus the applicability of existing data to the particular application.

However, there is no published information on the lowest hue discrimination thresholds of non-spectral colours (the "line of purples"), since these colours do not really have a dominant wavelength (they are the effect of a natural white colour lacking a specific wavelength around the green area). Therefore, another aspect of experimentation by the authors was concerned with establishing these discrimination thresholds. The initial expectation that the thresholds would be similar to the complementary (green) colours was verified experimentally.

Finally, the lowest saturation discrimination thresholds were established. More specifically, the authors measured the amount of pure hue needed to be added to white, mid-grey and black until the hue becomes detectable. All thresholds were experimentally derived, with the exception of the case of white for which existing biological information [18] was noted and verified. The applicability of the experimental results to non-spectral colours was also verified.

The above information on colour discrimination thresholds (as verified and extended by the authors) is utilised in each stage of the method described below in order to assess colour similarity.

\subsection{Chromaticlachromatic layer splitting}

The segmentation method begins with a necessary preprocessing step, which separates the chromatic from the 
achromatic content (pixels) of the image for independent further processing.

Chromatic colour is any colour for which a dominant wavelength can be identified (red, green, blue, yellow, etc). On the other hand, if no dominant wavelength can be identified, a colour is said to be achromatic (shades of grey, including black and white).

Separating chromatic from achromatic pixels at this stage is important as any process that examines Hue values will fail if applied to achromatic pixels, since the Hue for these pixels is either undefined, or unreliable [20].

The exact levels of Saturation and Lightness for which colours should be considered achromatic are not straightforward to set. The extended biological data prepared by the authors (see previous section) on the least noticeable differences in colour purity (saturation) are used here to define the border surface between chromatic and achromatic pixels in the HLS colour space. In Fig. 3 a slice of the HLS colour space is presented, where the marked centre area denotes the colours considered achromatic based on the measurements made. It should be noted that in the authors' experience this use of information derived from experiments in realistic situations (taking into account the effect of different Hues) is superior to using a single Saturation (and Lightness) threshold for separating chromatic from achromatic pixels (the border surface would have been depicted as a circle in Fig. 3).

Based on this information, the image is split in two layers, one containing all the achromatic pixels (perceived as grey-levels) and the other all the chromatic ones (see first level of the tree in Fig. 8). Characters may be present in either or both layers. Fig. 4 shows an example of an image in which some characters ("Forum" and "Boon or Bane?") are present wholly in the achromatic layer while others ("The U.S. in Panama") are wholly in the chromatic layer. Fig. 5 shows an instance of an image where parts of the same character lie in both the achromatic and the chromatic layers.

The chromatic layer is further processed based on both the Hue and Lightness components, whereas the achromatic layer is strictly kept out of any subsequent processing involving Hue.

\subsection{Further splitting}

This subsequent splitting process attempts to identify and describe areas of similar (as humans perceive it) colour in the image. To achieve this, the chromatic and achromatic layers are split into a number of more refined (in terms of colour uniformity) layers, based on global information
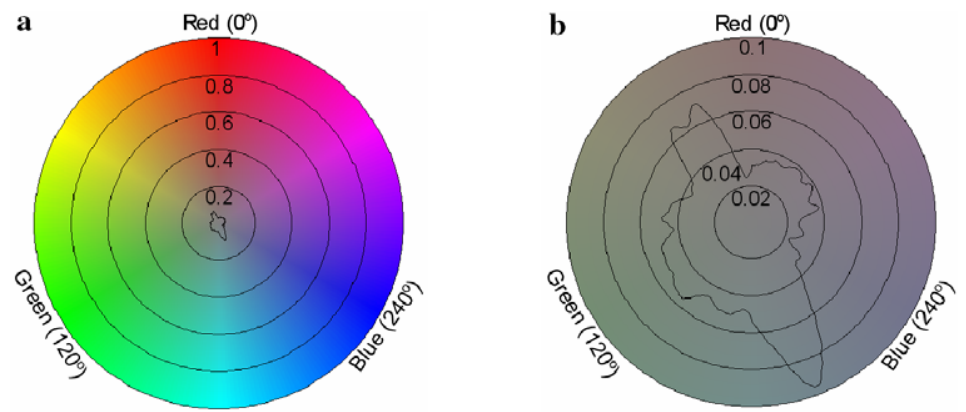

Fig. 3. (a) Outline of (perceived) chromatic/achromatic boundary surface shown through a slice at $L=128$, and (b) magnified.
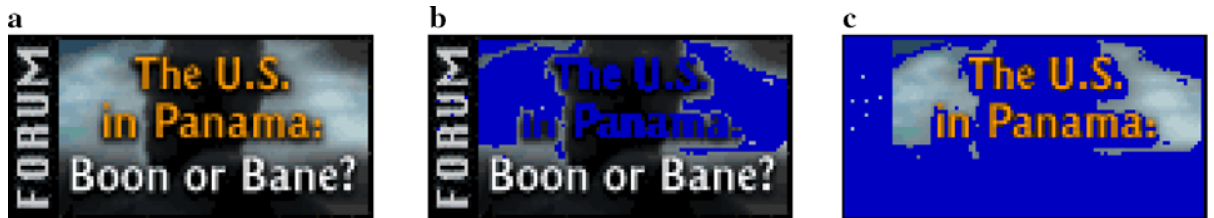

Fig. 4. Example of an image (a) where some characters are in the achromatic (b) and some in the chromatic (c) layer.
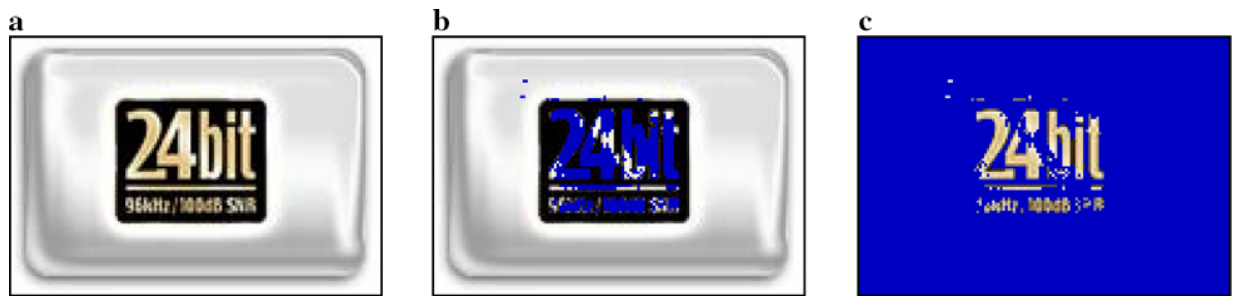

Fig. 5. Example of an image (a) where the same characters lie within both the achromatic (b) and the chromatic (c) layer. 
derived from the (Hue or Lightness, as appropriate) histogram of each layer.

\subsubsection{Achromatic layer}

For the pixels of the achromatic layer, the histogram of Lightness (the only available information) is computed and peaks are identified by locating minima and maxima. Each peak is described by a left-minimum, a maximum and a right-minimum. An example of a Lightness histogram can be seen at the lower part of Fig. 6 .

If two or more peaks are present the splitting process proceeds as follows, otherwise it stops (leaving the achromatic layer unchanged). Consecutive peaks are analysed by examining the horizontal distance (Lightness value difference) between them and their corresponding height difference (ratio of peak maxima).

Certain groups of (adjacent) peaks are combined if those peaks represent colours (shades) that are deemed to be perceived as 'similar' by a human observer. Similarity is considered and measured in two complementary ways using the two measurements mentioned above. The first, Lightness value similarity, is determined based on the results of the experiments designed and conducted by the authors, which established the least noticeable (by humans) lightness differences, as explained in Section 3.1. The second, the similarity of the height of the adjacent peaks (the ratio of peak maxima) under consideration is assessed to accommodate for the possible presence of gradient colour (as observed in experiments) [20]. At the end of this peak analysis and combination process, the pixels in the layer that have Lightness values under each final peak group, are exported to a separate sub-layer. Fig. 6 shows the achromatic layer of an image (root of the tree) and the corresponding Lightness histogram. The final (combined) peak groups are shown as intervals in the histogram and some of the corresponding sub-layers (for brevity) are illustrated as children of the achromatic layer.

The resulting sub-layers cannot be split further, since the only information available for achromatic pixels is their Lightness values. Therefore, the splitting process for the achromatic layer stops at this point.

\subsubsection{Chromatic layer}

For chromatic colours, the most important factors in terms of colour discrimination are mostly Hue and Lightness [17]. Consequently, there are two options to first split the chromatic layer: either based on the Hue histogram or based on the Lightness histogram. In that respect, previous research $[21,22]$ indicates that Hue has the greatest discrimination power among colour components and, more often than not, humans first differentiate objects according to their Hue, and then interpret differences in Lightness as shadows or highlights of the objects [22].

In view of the above, the histogram of the Hue values is first computed for the pixels of the chromatic layer and peaks are identified (in the same way as peaks of the Lightness histogram in the achromatic layer). Again, the horizontal distance (Hue difference) between consecutive peaks and their corresponding difference in height is calculated. Adjacent peaks are combined in this case if the Hue values spanned by the peaks are deemed to be perceived as 'similar' by a human observer. Similarity is assessed by examining both Hue similarity (defined based on the biological data discussed in Section 3.1) between peaks and the corresponding height ratio (to account for the presence of gradient colour). The chromatic layer is thus split into sub-layers of different Hue (each layer containing the range of hues under each of the final peaks). An example of chromatic layer splitting based on the Hue histogram is shown in Fig. 7. The chromatic layer of the image (root) gives rise
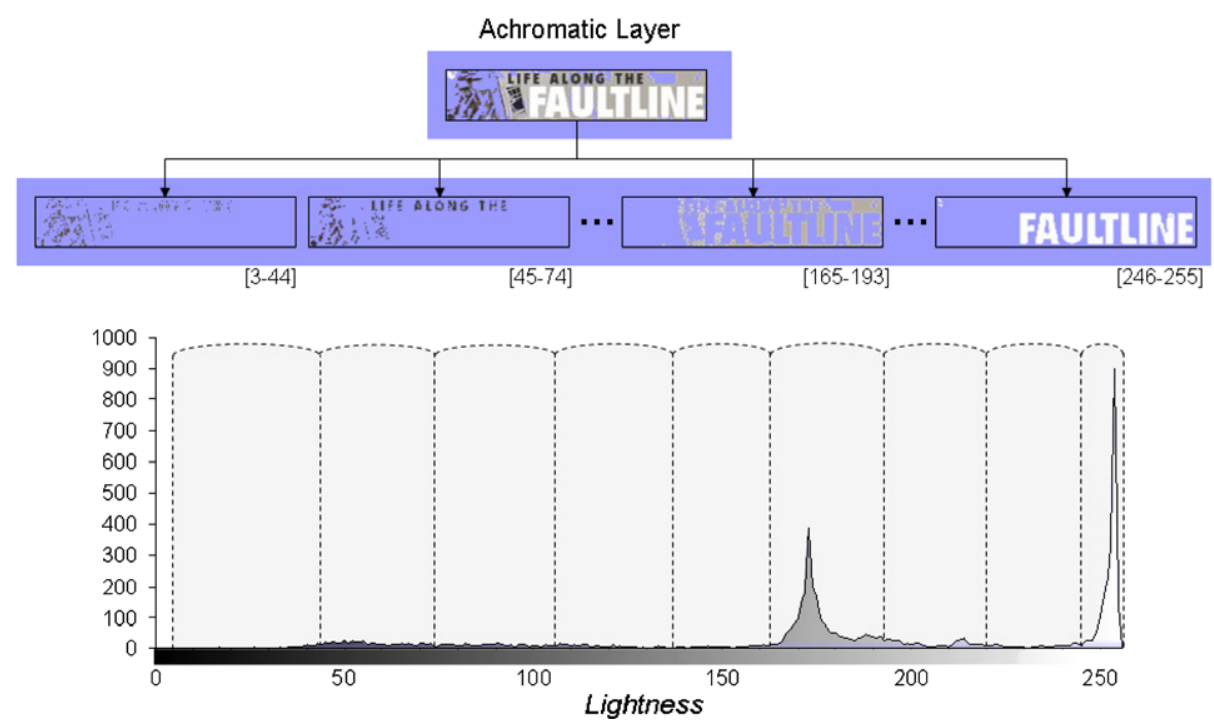

Fig. 6. Achromatic layer of an image and some of the Lightness sub-layers produced. The Lightness histogram of the achromatic layer is shown and the groups of peaks (resulting in Lightness sub-layers) indicated. 


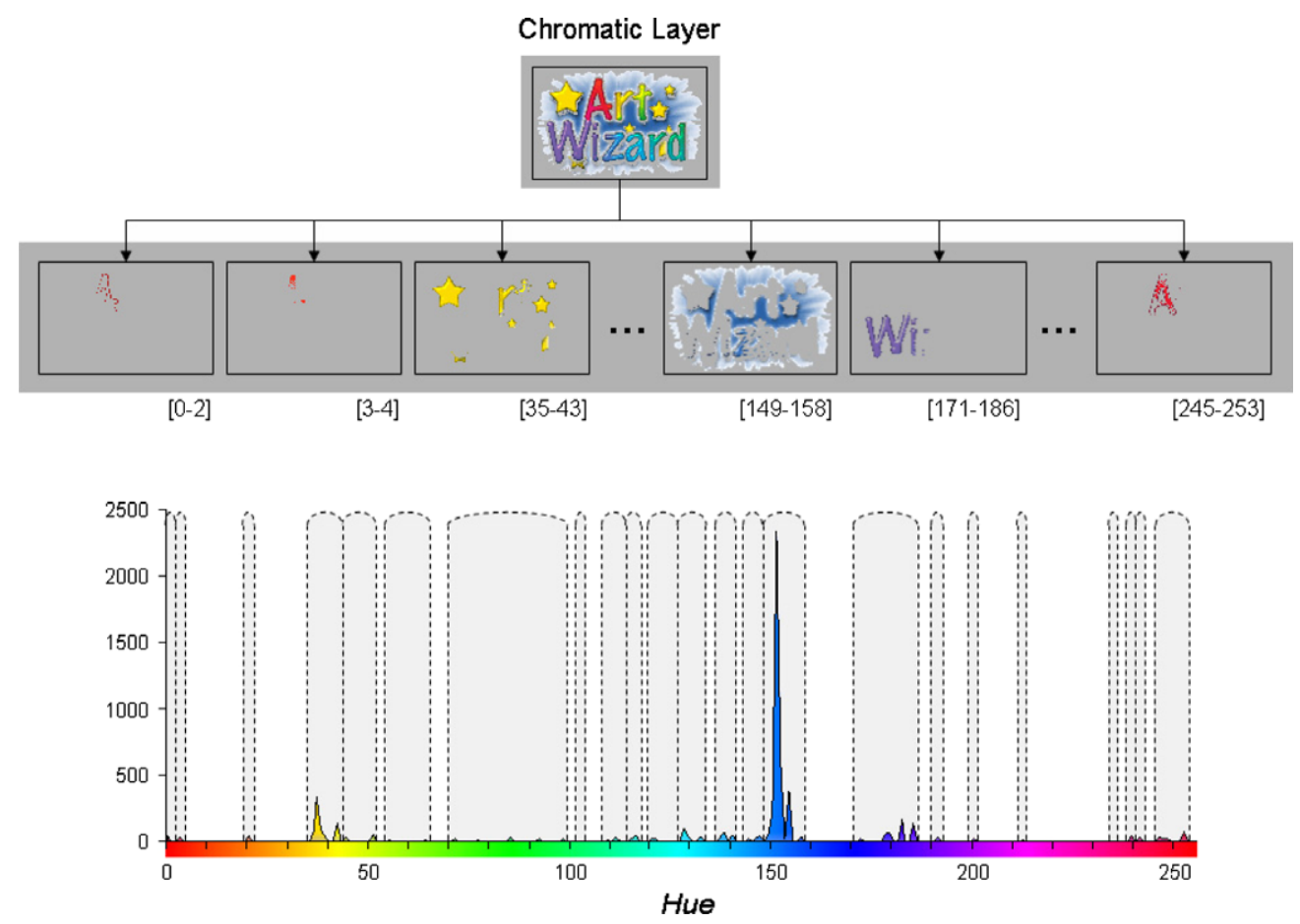

Fig. 7. Chromatic layer of an image and part of the Hue sub-layers produced. The Hue histogram of the chromatic layer is shown and the groups of peaks (resulting in Hue sub-layers) indicated.

to the histogram at the lower part of the figure. Note that the absence of certain hues is manifested by a discontinuous histogram. Groups of combined peaks are indicated by intervals (for illustration purposes). The sub-layers produced for some of these intervals are shown as children of the chromatic layer.

For each of the sub-layers produced, the Lightness histogram is then computed, peaks are identified and the peak analysis and grouping process is performed (as in the achromatic layer). New image sub-layers are created for pixels with Lightness values in the ranges under each of the final peak groups.

It should be noted that the splitting process is terminated early if only a single peak is identified in the histogram currently analysed (Hue or Lightness). In that case the corresponding layer remains unchanged (not split).

\subsubsection{Layer tree}

Following the splitting of the image into achromatic and chromatic layers and their corresponding sub-layers, a tree representation is produced. The root represents the original image and the nodes correspond to each of the layers produced by the splitting process. Each layer contains regions of a certain distinct (to humans) Hue or Lightness. Fig. 8 illustrates the layer tree representation.

\subsection{Colour connected component information}

At this point, each bottom (leaf) layer contains a subset of the pixels of the original image, as filtered through the (successive) splitting (i.e., the union of all leaf layers is the original image). In order to prepare for possible merging (Section 3.5), connected components are identified on each leaf layer, using a one-pass labelling algorithm [23] and the average colour of each component is computed and stored.

Each connected component corresponds to a region in the image that has an effectively distinct (to humans) colour. In terms of characters, each connected component may correspond to a single character but, more often than not, it corresponds to part of a character or stroke. It should be noted at this point that over-splitting of characters occurs regularly due to the cautious nature of the splitting process (the similarity criteria are strictly enforced regarding the separation of colours that are perceived to be even slightly different). In contrast, the subsequent merging process (Section 3.5) is based on a more relaxed colour similarity principle (components whose colour may be perceived as 'slightly' different are still merged) and is also guided by local evidence. The features (characteristics) used and their derivation are explained next.

\subsubsection{Vexed area}

The principle of component extensibility is examined first, based on colour similarity. For each component in each leaf layer, its neighbourhood in the original image is analysed to determine which pixels could be annexed to the component based on colour similarity. The pixels comprising that potential extension of that region are collectively referred to as the vexed area of the region (see the blue areas of the two components in Fig. 9). 


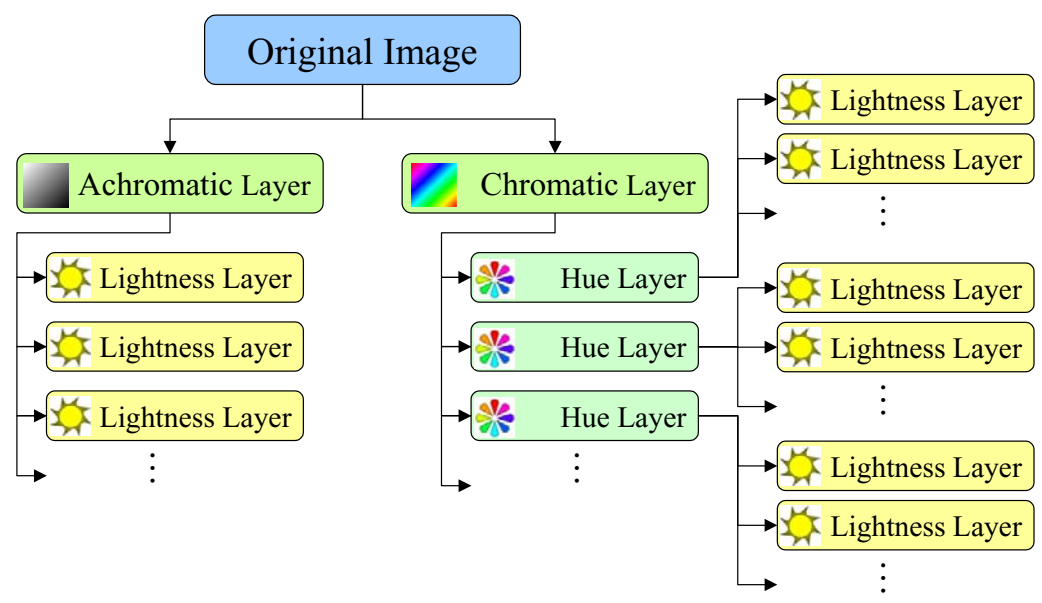

Fig. 8. The tree structure of layers produced by splitting.
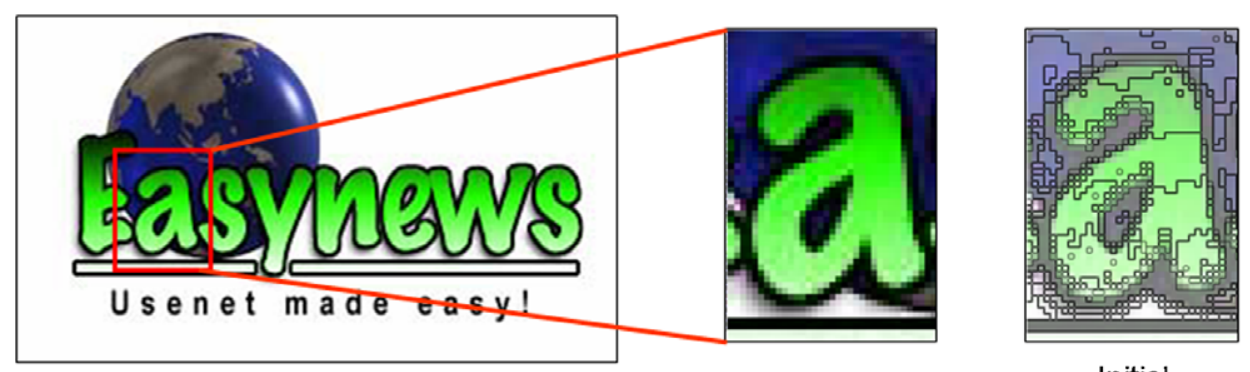

Initial Segmentation
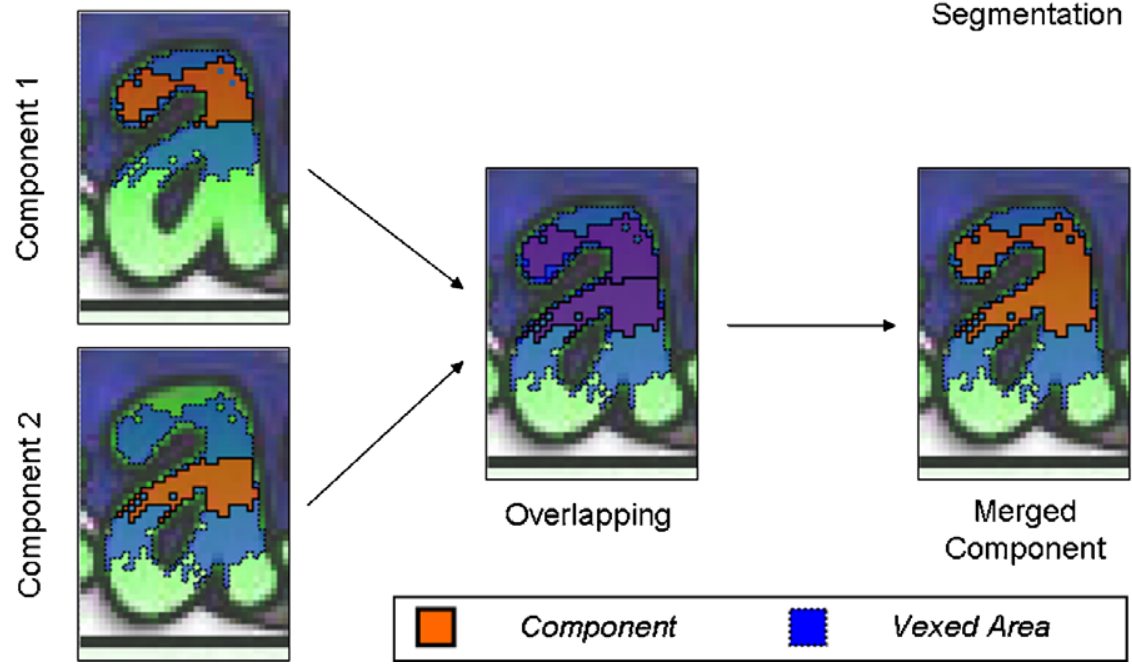

Fig. 9. Merging process. Starting from the connected components identified in the leaf layers (Initial Segmentation) pairs of components and their vexed areas are examined. If the overlapping degree is more than a specified threshold the components are merged to form a single component with a vexed area defined as the combination of the original vexed areas.

The overarching idea is that a human should not be able to perceive a significant difference between the core region (component) and the vexed area pixels. For layers resulting from splitting based on the Hue histogram, the analysis criterion corresponds to humans' minimum colour discrimination ability as a function of wavelength. For layers resulting from splitting based on the Lightness histogram the criterion corresponds to humans' minimum colour discrimination ability as a function of lightness.
The thresholds for the colour similarity criteria have been experimentally determined based on the biological data (with respect to wavelength and lightness) explained earlier. It should be noted, however, that there is a significant difference in the use of this biological data. In the determination of vexed areas the thresholds are relatively relaxed as the emphasis is on collecting all pixels that a human will perceive as similar. In contrast, in the splitting stage the emphasis was on strict distinction 
between any pixels that could be perceived as being different.

After this examination of the neighbourhood of each component, all pixels that fulfil the relevant colour similarity criterion are noted as the vexed area of that component.

\subsubsection{Overlapping}

In addition to component extensibility (determination of vexed areas) as discussed above, the topological relationship between components is examined. This leads to the need for an expression of overlap between two components (taking also into account their vexed areas).

An expression of overlap is derived as follows: Given two components $a$ and $b$, and their associated vexed areas $a_{\mathrm{v}}$ and $b_{\mathrm{v}}, N C P\left(a_{\mathrm{v}}, b\right)$ is the number of common pixels between the vexed area of component $a$ and component $b$, and $N C P\left(a, b_{\mathrm{v}}\right)$ is the number of common pixels between component $a$ and the vexed area of component $b$. Therefore, $N C P\left(a_{\mathrm{v}}, b\right)=\left|a_{\mathrm{v}} \cap b\right|$ and $N C P\left(a, b_{\mathrm{v}}\right)=\left|a \cap b_{\mathrm{v}}\right|$.

The overlapping between components $a$ and $b$, denoted $\operatorname{Ovl}(a, b)$, is defined as in Eq. (1), as the number of overlapping pixels between the components $a$ and $b$, given by $N C P\left(a_{\mathrm{v}}, b\right)+N C P\left(a, b_{\mathrm{v}}\right)$, divided by the maximum number of pixels that could potentially overlap.

$\operatorname{Ovl}(a, b)=\frac{N C P\left(a_{\mathrm{v}}, b\right)+N C P\left(a, b_{\mathrm{v}}\right)}{\min \left(\text { Area }\left(a_{\mathrm{v}}\right), \operatorname{Area}(b)\right)+\min \left(\operatorname{Area}(a), \operatorname{Area}\left(b_{\mathrm{v}}\right)\right)}$

where $\operatorname{Area}(x)$ denotes the number of pixels of $x$.

Detailed experiments carried out by the authors have shown that $\operatorname{Ovl}(a, b)$ gives a reliable indication of the suitability of the overlap (for the purpose of determining and prioritising a possible merger - see below). However, it is less reliable in special cases where the areas of the components involved are comparable to the number of pixels overlapping: $N C P\left(a_{\mathrm{v}}, b\right)+N C P\left(a, b_{\mathrm{v}}\right)$. To take this into account a suitable weighting function was experimentally derived [20]:

$W(a, b)=\frac{N C P\left(a_{\mathrm{v}}, b\right)+N C P\left(a, b_{\mathrm{v}}\right)}{2 \cdot \min (\operatorname{Area}(a), \text { Area }(b))}$

The weighted overlapping, referred to from here onward as the overlapping degree, for a pair of components $a$ and $b$, $W(a, b) \cdot \operatorname{Ovl}(a, b)$, is the feature finally used. For each pair of components, the overlapping degree is computed and stored.

\subsection{Merging strategy}

In each of the leaf layers, every possible combination of components is examined, and, if their overlapping degree is above a pre-defined threshold (experimentally derived: 0.56 ), a possible merger is identified. All identified possible mergers in each layer are kept in a sorted list and merging starts with the pair of components having the highest overlapping degree. When two components $a$ and $b$ are merged, the two core regions are combined to form the core region of the new component $c$ (i.e., $c=a \cup b$ ). The vexed area $c_{\mathrm{v}}$ of the new core component will be the remainder of the vexed areas of $a$ and $b$ (i.e., $c_{\mathrm{v}}=\left\{p \mid p \in\left(a_{v} \cup b_{\mathrm{v}} \wedge\right.\right.$ $p \notin a \cup b\})$. An illustration of the merger of two components is shown in Fig. 9. First, the initial segmentation (region contours) is indicated. Two components that form part of the character and their corresponding vexed areas are shown next. The overlap of core regions and vexed areas is shown for the two components (indicated as blended colours). Finally, the newly created component is presented with its vexed area.

After each merger is completed, other potential mergers involving one of the two no-longer-existing components $a$ and $b$ with an existing component, e.g., $k$, are reassessed to take into account the resulting newly created component, $c$. The overlapping degree between $k$ and $c$ is calculated and the list of potential mergers is suitably updated. The process continues as long as there are potential mergers in the list with overlapping degree above the threshold (as described in the beginning of this section).

After all possible mergers have taken place within each of the leaf layers, merging between the components of all sibling layers (across the same level) is performed. This involves copying the final components of the leaf layers one level up, and repeating the merging process in the layer that receives the components. At the end of the merging process in that parent layer, the vexed areas of the resulting components need to be refined, so that they are representative of the new layer in which they now reside (the vexed areas of the components were identified in the leaf layers, according to the type of those leaf layers). For instance, after copying all the components identified in the Lightness leaf layers to their parent Hue layer, the vexed areas must be refined so that they contain pixels not only of similar Lightness to the component, but of similar Hue as well. This is important, as merging between sibling Hue layers (at the same tree level) will be performed next, and this merging must be based on Hue similarities.

After the refinement of vexed areas a component integrity check is performed. The purpose of this process is to assess the validity of components resulting from the previous merging activity and, if necessary, to dismantle merged components that are unlikely to represent parts of characters. This situation may happen when at the end of all possible mergers there exist components that are disjoint (the union of the merged components is not a single connected component). In such a case, the disjoint components are relabelled as separate components.

The merging process is repeated level by level, moving up in the tree structure until the root of the tree (the original image) is reached. More specifically, all connected components created in layers at the same level in the tree structure are copied one level up. At that (higher) level the following sequence of steps takes place: merging, refinement of the vexed areas (according to the type of that layer) 


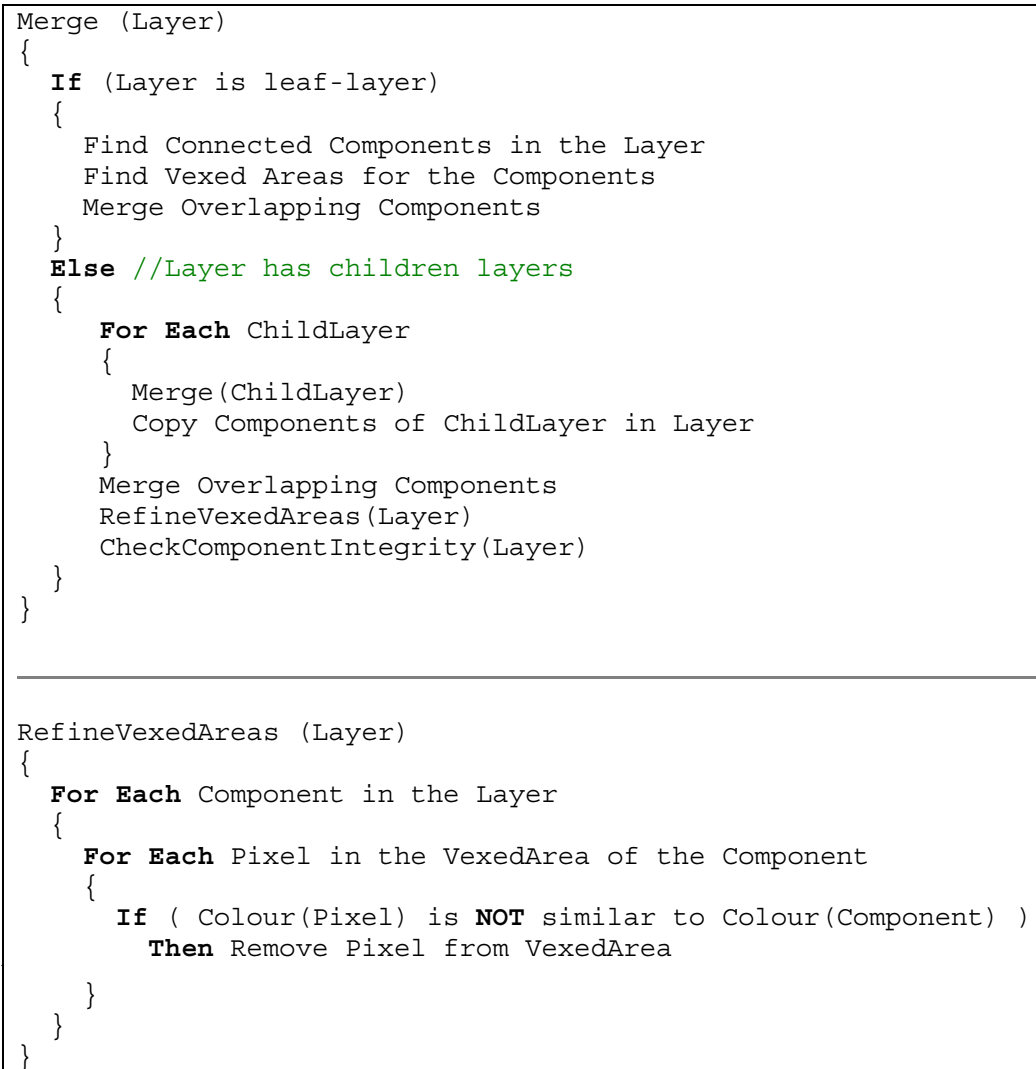

Fig. 10. Pseudo-code describing the merging process.

and component integrity checking. The resulting components are then copied to the next higher level. An illustration of the process is given in the form of pseudo-code in Fig. 10.

At the end of the final merging process in the top-level (root) layer, the original image has been segmented into a number of connected components, some of which will correspond to characters (or character fragments) and the rest will be part of the background (everything which is not text).

The character segmentation stage is now complete and forms the basis for further processes towards the extraction and recognition of text. A detailed evaluation of the complete segmentation process is given below (Section 4), followed by an examination of the possibilities offered beyond segmentation.

\section{Evaluation}

The degree of success of any process that follows segmentation will heavily depend on how well the background is separated from the characters. As mentioned in the Introduction, the character segmentation process is both crucial to the performance of subsequent processes and has to deal with certain challenges that are not necessarily faced by extraction and recognition. As such, the character segmentation results constitute a milestone that warrants individual study and evaluation, without the additional, possibly application-specific stages that introduce their own errors.

The performance of the segmentation method has been evaluated using a dataset created by the authors. Significant attention has been paid to design the dataset to be as representative as (realistically) possible of the variations of text in image form present on the Web. To that effect, two issues play an important role in the selection of images. First, the size of the dataset is determined by balancing the specific evaluation needs with the difficulty in its creation. For segmentation evaluation (as opposed to OCR or text retrieval evaluation, for instance) a relatively compact dataset is generally sufficient in representing the difficulties at the pixel level (a consideration shared by previous approaches as well $[3,9])$.

Second, the selection of the images must be based on both technical difficulty (to represent a variety of conditions) and context of use criteria (to reflect a realistic distribution of the different levels of difficulty). The dataset focuses on images containing text that a typical user may encounter on a Web document they will find while fulfilling a typical everyday request. Images containing text on websites of newspapers, companies, academic sites, e-commerce sites, search engines etc. were included in the sample. The function (header, menu item, logo, equation etc.) of an occurrence of image text in the corresponding Web document was also considered when 
creating the dataset, so that the resulting collection is representative in this respect as well.

Furthermore, text in the images of the dataset is characterized as either readable or non-readable. A minimum size (width of 4 pixels and height of 6 pixels) was determined for a character to be considered readable, since even humans have difficulties recognizing characters smaller than this threshold (see Fig. 11(a)). Other issues that affect readability (by humans) are the choice of colours and the use of anti-aliasing (see Fig. 11(b)). The classification of characters was made by a human operator, on a case-by-case basis.

The dataset comprises 115 images with varying degrees of complexity. The number of characters in the images ranges from 2 to 83 . On average, an image was found to have around 20 characters, out of which around 16 are readable. In total, the images in the dataset contain 2404 characters, out of which 1852 are classified as readable and 552 are classified as non-readable.

Finally, the images in the dataset were grouped into four categories according to the colour combinations of text and background. Category $A$ holds images that contain multicolour characters over multicolour background (14 images). Category $B$ contains images that have multicolour characters over single-colour background (15 images). Category $C$ has images with single-colour characters over multicolour background (37 images). Finally, Category $D$ holds images with single-colour characters rendered over single-colour background (49 images). The distribution of images in the four categories reflects the occurrence of images of those types on Web documents.

The evaluation of the segmentation method was performed by visual inspection of the results produced on all the images in the dataset (similarly to previous approaches). Automated evaluation was considered but was not possible in this case for two reasons. First, the authors consider that the use of synthetic data will not be representative enough of the situations under evaluation. Second, there is no ground truth available for real Web images (it is actually not straightforward to create accurate segmentation ground truth for such images [20]).

Each character contained in the segmented image is characterised as identified, split, merged or missed. Identified characters are those that are described by a single component. As split are considered the characters described by more than one component, as long as each of those compo- nents contain only pixels of the character in question (not any additional background or other character pixels). If two or more characters are described by only one component (which does not contain any non-character pixels), then they are considered as merged. Finally, missed are the characters for which no component or combination of components exists that describes them completely without containing non-character pixels.

In practical terms, any segmentation where a character is not "missed" is useful for further processing, even if the character is "merged" or "split". Furthermore, it should be noted that the above characterisation of segmentation results is very strict in, effectively, considering any character that contains non-character pixels as wrong. This is a conscious choice, however, to assess the absolute performance of the segmentation method without including any components that could potentially pose problems to OCR.

The overall results for the split and merged method can be seen in Table 2. In total, the method was able to correctly identify $1290(69.65 \%)$ out of 1852 readable characters, while $151(8.15 \%)$ characters were merged, $271(14.63 \%)$ were split and $140(7.56 \%)$ characters were missed. In addition, out of the 552 non-readable characters, the method was able to identify $184(33.33 \%)$.

Examples of representative images from the dataset and the corresponding results are shown in Fig. 12. In the results, characters in black colour have been identified, characters in blue colour have been merged, and characters in red have been split (however, no background parts have been merged with characters).

As expected, the method worked better for images in categories $\mathrm{C}$ and $\mathrm{D}$ - i.e., images containing text of single colour. However, considering the complexity encountered, it also performed satisfactorily well on images in categories $\mathrm{A}$ and $\mathrm{B}$ - i.e., those containing multicoloured text. It should be mentioned that over-splitting the image into layers, does not seem to affect the merging process, which is able to combine (reconstitute) the oversplit components, so long as their fragments are visually similar. Naturally, the overall results reflect the increasing difficulty in categories where the text and/or the background are multicoloured.

It is not very meaningful to compare directly the results of this method to existing ones as, in contrast to those, the

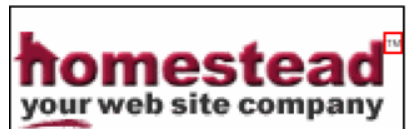

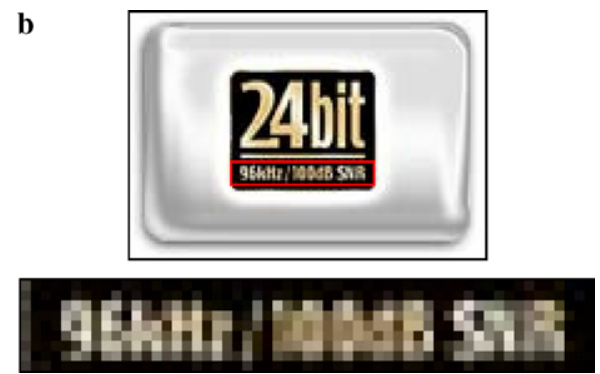

Fig. 11. Examples of non-readable text (a) too small, (b) badly designed in terms of colours and excessive anti-aliasing. 
Table 2

Results of the split and merge method over all images of the dataset

\begin{tabular}{|c|c|c|c|c|c|c|}
\hline & & Number of characters & Identified & Merged & Split & Missed \\
\hline \multirow[t]{2}{*}{ All categories } & Readable & 1852 & $1290(69.65 \%)$ & $151(8.15 \%)$ & $271(14.63 \%)$ & $140(7.56 \%)$ \\
\hline & Non-readable & 552 & $184(33.33 \%)$ & $22(3.99 \%)$ & $160(28.99 \%)$ & $186(33.70 \%)$ \\
\hline \multirow[t]{2}{*}{ Category A } & Readable & 206 & $115(55.83 \%)$ & $0(0.00 \%)$ & $60(29.13 \%)$ & $31(15.05 \%)$ \\
\hline & Non-readable & 58 & $12(20.69 \%)$ & $2(3.45 \%)$ & $15(25.86 \%)$ & $29(50.00 \%)$ \\
\hline \multirow[t]{2}{*}{ Category B } & Readable & 260 & $135(51.92 \%)$ & $48(18.46 \%)$ & $67(25.77 \%)$ & $10(3.85 \%)$ \\
\hline & Non-readable & 42 & $6(14.29 \%)$ & $3(7.14 \%)$ & $3(7.14 \%)$ & $30(71.43 \%)$ \\
\hline \multirow[t]{2}{*}{ Category $\mathrm{C}$} & Readable & 699 & $530(75.82 \%)$ & $48(6.87 \%)$ & $64(9.16 \%)$ & $57(8.15 \%)$ \\
\hline & Non-readable & 150 & $55(36.67 \%)$ & $11(7.33 \%)$ & $45(30.00 \%)$ & $39(26.00 \%)$ \\
\hline \multirow[t]{2}{*}{ Category D } & Readable & 687 & $510(74.24 \%)$ & $55(8.01 \%)$ & $80(11.64 \%)$ & $42(6.11 \%)$ \\
\hline & Non-readable & 302 & $111(36.75 \%)$ & $6(1.99 \%)$ & $97(32.12 \%)$ & $88(29.14 \%)$ \\
\hline
\end{tabular}

a

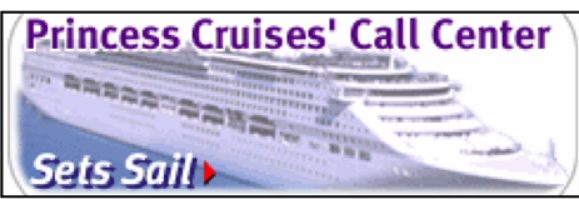

b

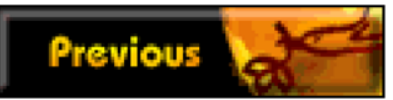

c

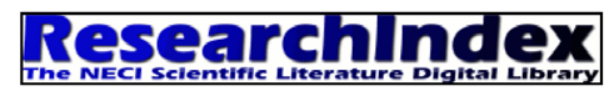

d

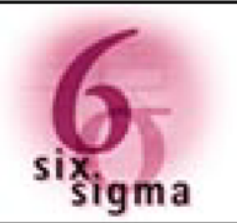

e

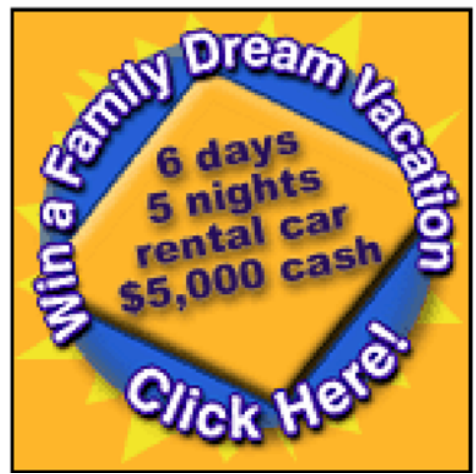

Princess Cruises' Call Center

Sets Soil

Previous

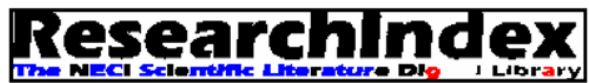

Whe NECI Selemente Uterreture Dlo, Library
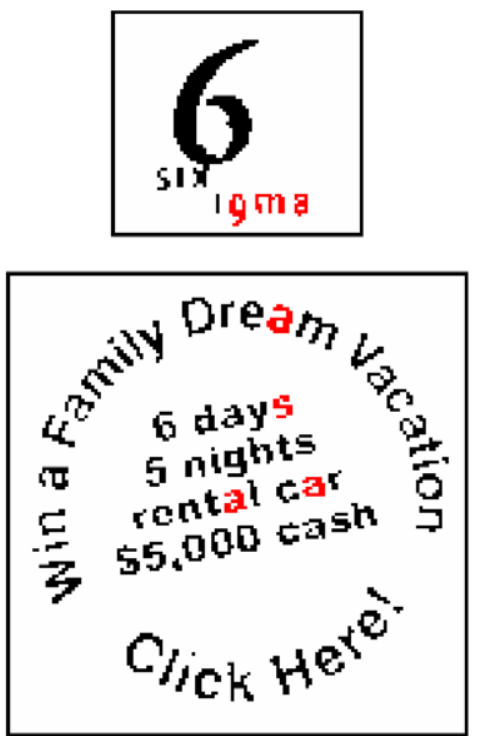

Fig. 12. A number of representative images with the corresponding results.

proposed approach is applicable to a much broader range of character images that pose widely varying levels of difficulty.

More specifically, of the very few methods dedicated to the analysis of text in web images, detailed results are only given in the work of Lopresti and Zhou [3]. As described in Section 2, that method is designed for (and consequently evaluated on) GIF images, which contain a maximum of 256 colours only. Moreover, the assessment of that method was concentrated on a subgroup of Web images that meet the assumptions set for that method (the test images contain homogeneously coloured text). For that specific type of image, a detection rate of $78.8 \%$ is reported. The authors of that method also report a relatively low character detection rate $(36.6 \%)$ on a very small set of images in which the character and the background colours are not homogeneous. Similarly, for very small characters (in the 'unreadable' category in the context of this method), a low detection rate of $44.3 \%$ is reported. 
Bearing in mind that a direct comparison between the method of Lopresti and Zhou [3] and the proposed one is not very meaningful, in broad terms the performance of the two methods on the homogeneous images is comparable. However, the proposed approach is also applicable to the very wide range of images found on the Web and in this respect it is superior to previously published methods.

\section{Beyond character segmentation}

This section explores concrete ways of exploiting the results of character segmentation within the context of Web image text recognition. To demonstrate a viable extension of the character segmentation process, the authors have developed a test application to identify textlike groups of connected components [20]. The objective is to extract sets of connected components that satisfy criteria for being part of text (without any attempt to recognise the text).

It must be noted that there can be several approaches to the extraction and recognition of the text based on the same segmentation results. These can range from simple connected component analysis and OCR to integrated approaches involving feedback from recognition and the utilisation of contextual information. The demonstrator approach described here is relatively simple and is based on geometric criteria only.

The method exploits the fact that characters belonging to the same word or textline share common characteristics, e.g., they are similarly sized and lie on the same baseline (not necessarily a straight line), and attempts to identify such groups of connected components. The components are first grouped according to their size and an attempt is made to identify straight lines of components in each size group. For each line identified an assessment process follows, which indicates whether it is a valid textline or not.

The assessment process involves two scoring mechanisms. One mechanism examines the distances between successive components in a candidate (identified in the previous step) line, and assigns a higher confidence value to lines whose components have equal distances between them. The other mechanism devised for assessing the lines, uses the projection profile of the components along the direction of the line identified, and examines whether this projection is structurally similar to the projection profile expected from a real textline. Lines yielding a score above a specified confidence threshold (defined as part of the scoring scheme) are output as true character lines.

The method is able to identify lines (straight or curving) of components corresponding to characters in most of the cases as long as a good segmentation has been obtained. This highlights the importance of the character segmentation stage to the text extraction process.

The most frequent problem encountered is the wrongful inclusion (in the extracted text-like lines) of components which do not actually correspond to characters. A possible solution to this issue would be to incorporate additional component filtering using further character properties (post processing steps like this are suggested as future work). It should be noted, however, that this type of error further highlights that the character segmentation results are indeed useful and that the segmentation method is successful.

\section{Concluding remarks}

This paper has presented a novel method to the topical problem of segmenting characters in colour Web images containing text (headers, titles, banners etc. that are often artistically created). In contrast to previous approaches, this paper abandons analysis in the RGB colour space and adopts a segmentation method based on analysing differences in chromaticity and lightness that is closer to how humans perceive distinct objects. Human perception-specific characteristics of the method were presented and discussed and the segmentation method was detailed and evaluated. Results show a superior ability of the method to segment characters in complex situations such as in the presence of varying colour and texture (characters and background).

\section{References}

[1] Search Engine Watch, http://searchenginewatch.com.

[2] A. Antonacopoulos, D. Karatzas, J. Ortiz Lopez, Accessing textual information embedded in internet images, in: Proceedings of SPIE Internet Imaging II, San Jose, USA, January 24-26, 2001, pp. 198205.

[3] D. Lopresti, J. Zhou, Locating and recognizing text in WWW images, Inf. Retrieval 2 (2000) 177-206.

[4] M.K. Brown, Web page analysis for voice browsing, in: Proceedings of the 1st International Workshop on Web Document Analysis (WDA '2001), Seattle, USA, September 2001, pp. 59-61.

[5] G. Penn, J. Hu, H. Luo, R. McDonald, Flexible web document analysis for delivery to narrow-bandwidth devices, in: Proceedings of the 6th International Conference on Document Analysis and Recognition (ICDAR'01), Seattle, USA, September 2001, pp. 1074-1078.

[6] A. Antonacopoulos, J. Hu (Eds.), Web Document Analysis: Challenges and Opportunities, Series in Machine Perception and Artificial Intelligence, World Scientific Publishing Company, New York, 2003.

[7] A. Antonacopoulos, D. Karatzas, An anthropocentric approach to text extraction from WWW images, in: Proceedings of the 4th IAPR International Workshop on Document Analysis Systems (DAS2000), Rio de Janeiro, Brazil, December 2000, pp. 515-525.

[8] A. Antonacopoulos, F. Delporte, Automated interpretation of visual representations: extracting textual information from WWW images, in: R. Paton, I. Neilson (Eds.), Visual Representations and Interpretations, Springer, London, 1999.

[9] A.K. Jain, B. Yu, Automatic text location in images and video frames, Pattern Recognit. 31 (12) (1998) 2055-2076.

[10] R. Lienhart, F. Stuber, Automatic text recognition in digital videos, in: Proceedings of SPIE Volume: 2666 - Image and Video Processing IV, 1996, pp. 180-188.

[11] H.K. Kim, Efficient automatic text location method and contentbased indexing and structuring of video database, J. Vis. Commun. Image Represent. 7 (1996) 336-344.

[12] V. Wu, R. Manmatha, E.M. Riseman, Finding text in images, in: Proceedings of the 2nd ACM International Conference on Digital Libraries, Philadelphia, PA, 1997, pp. 23-26. 


\section{ARTICLE IN PRESS}

[13] P. Clark, M. Mirmehdi, Recognising text in real scenes, Int. J. Doc. Anal. Recognit. 4 (2002) 243-257.

[14] S. Messelodi, C.M. Modena, Automatic identification and skew estimation of text lines in real scene images, Pattern Recognit. 32 (1999) 791-810.

[15] R.S. Ledley, M. Buas, T.J. Golab, Fundamentals of true-color image processing, in: Proceedings of the 10th International Conference on Pattern Recognition, Atlantic City, New Jersey, June 1990, pp. 791795.

[16] S. Tominaga, Color image segmentation using three perceptual attributes, in: Proceedings of the Conference on Computer Vision and Pattern Recognition, Miami, Florida, 1986, pp.628-630.

[17] L.D. Silverstein, Human factors for color display systems: concepts, methods and research, in: H.J. Durrett (Ed.), Color and the Computer, Academic Press, Orlando, Florida, 1987, pp. 27-61.
[18] G. Wyszecki, W. Stiles, Color Science: Concepts and Methods, Quantitative Data and Formulae, 2e, Wiley, New York, 1982.

[19] R.E. Bedford, G.W. Wyszecki, Wavelength discrimination for point sources, J. Opt. Soc. Am. 48 (2) (1958).

[20] D. Karatzas, Text segmentation in web images using colour perception and topological features, PhD Thesis, University of Liverpool, UK, 2002

[21] A.R. Weeks, G.E. Hague, Color segmentation in the his color space using the K-means algorithm, in: Proceedings of SPIE Volume: 3026 - Nonlinear Image Processing VIII, 1997, pp. 143-154.

[22] A. Moghaddamzadeh, N. Bourbakis, A fuzzy region growing approach for segmentation of color images, Pattern Recognit. 30 (1997) 867-881.

[23] A. Antonacopoulos, Page segmentation using the description of the background, Comput. Vis. Image Underst. 70 (1998) 350-369. 